\title{
Sinonímias e novas combinações em Trachyderini e Acanthoderini (Coleoptera, Cerambycidae)
}

\author{
Miguel A. Monné1,2 \& Marcela L. Monné
}

${ }^{1}$ Museu Nacional, Universidade Federal do Rio de Janeiro. Quinta da Boa Vista, São Cristovão, 20940-040 Rio de Janeiro-RJ, Brasil. ${ }^{2}$ Bolsista do CNPq.

\begin{abstract}
Synonymies and new combinations in Trachyderini and Acanthoderini (Coleoptera, Cerambycidae). New synonymies: Lissonomimus Viana \& Martínez, 1992 = Laneiella Monné, 2006 syn. nov.; Acanthoderes latevittata Aurivillius, 1921 = Acanthoderes onca Galileo \& Martins, 2006 syn. nov.; Acanthoderes thoracicus White, $1855=$ Acanthoderes (Psapharochrus) uyapensis Martins \& Galileo, 2003 syn. nov.. Irundisaua Martins \& Galileo, 2005 is considered a synonym of Natagaima but the name Natagaima Lane, 1972 is preoccupied by Natagaima Beier, 1960 (Orthoptera, Tettigoniidae), we propose the substitution by the junior synonym Irundisaua Martins \& Galileo, 2005. New combinations: Lissonomimus megaderinus (Lane, 1973) comb. nov.; Irundisaua balteata (Lane, 1972) comb. nov.; Irundisaua heloisae (Julio, 2003) comb. nov.; Irundisaua moacyri (Julio, 2003) comb. nov..
\end{abstract}

KEYWORDS. Acanthoderes; Cerambycinae; Irundisaua; Lamiinae; Lissonomimus.

RESUMO. Sinonímias e novas combinações em Trachyderini e Acanthoderini (Coleoptera, Cerambycidae). Novas sinonímias: Lissonomimus Viana \& Martínez, 1992 = Laneiella Monné, 2006 syn. nov.; Acanthoderes latevittata Aurivillius, 1921 = Acanthoderes onca Galileo \& Martins, 2006 syn. nov.; Acanthoderes thoracicus White, $1855=$ Acanthoderes (Psapharochrus) uyapensis Martins \& Galileo, 2003 syn. nov.. Irundisaua Martins \& Galileo, 2005 é considerado sinônimo de Natagaima mas o nome Natagaima Lane, 1972 está preocupado por Natagaima Beier, 1960 (Orthoptera, Tettigoniidae), propomos a substituição pelo sinônimo júnior Irundisaua Martins \& Galileo, 2005. Novas combinações: Lissonomimus megaderinus (Lane, 1973) comb. nov.; Irundisaua balteata (Lane, 1972) comb. nov.; Irundisaua heloisae (Julio, 2003) comb. nov.; Irundisaua moacyri (Julio, 2003) comb. nov.

PALAVRAS-CHAVE. Acanthoderes; Cerambycinae; Irundisaua; Lamiinae; Lissonomimus.

São propostas quatro sinonímias e quatro novas combinações em Trachyderini (Cerambycinae) e Acanthoderini (Lamiinae). O material examinado pertence ao Museu Nacional, Universidade Federal do Rio de Janeiro, Rio de Janeiro (MNRJ).

Cerambycinae, Trachyderini

\section{Lissonomimus Viana \& Martínez, 1992}

Pujolia Lane, 1973: 380; Monné \& Monné, 1998: 533 (sin.); Monné, 2005a: 636 (cat.).

Lissonomimus Viana \& Martínez, 1992: 28; Monné, 2005a: 636 (cat.; in syn.).

Laneiella Monné, 2006: 179 (cat.) (nom. nov. pro Pujolia Lane non Pujolia Levasseur, 1968). Syn. nov.

Monné \& Monné (1998) propuseram a sinonímia entre Pujolia Lane, 1973 e Lissonomimus Viana \& Martínez, 1992. Monné (2006) criou desnecessariamente o nome Laneiella para substituir Pujolia Lane, 1973, preocupado por Pujolia Levasseur, 1968 (Coleoptera, Staphylinidae). Propomos a utilização do sinônimo júnior Lissonomimus Viana \& Martínez, 1992.

\section{Lissonomimus auratopilosus Di Iorio, 1998}

Lissonomimus auratopilosus Di Iorio, 1998: 28, figs. 1, 3.

Pujolia auratopilosa; Di Iorio, 2002: 76; Monné, 2005a: 636 (cat.).

Localidade-tipo: Argentina, Chaco: Charata. Distribuição: Argentina (Chaco).

A combinação original de Di Iorio (1998) é restaurada.

Lissonomimus megaderinus (Lane, 1973) comb. nov.

Pujolia megaderina Lane, 1973: 382; Monné \& Monné, 1998: 533 (sin.); Monné, 2005a: 637 (cat.).

Lissonomimus narcisoi Viana \& Martínez, 1992: 28, fig. 2; Di Iorio, 1998: 34.

Localidade-tipo: Brasil, Goiás: Jataí. Distribuição: Brasil (Goiás), Paraguai.

Lamiinae, Acanthoderini

Acanthoderes latevittata Aurivillius, 1921

Acanthoderes latevittata Aurivillius, 1921: 51, pl. 2, fig. 9.

Localidade-tipo: Bolívia. Distribuição: Bolívia. 
Acanthoderes (Psapharochrus) latevittata; Aurivillius, 1923: 386 (cat.). Psapharochrus latevittatus; Monné, 2005b: 207 (cat.).

Acanthoderes onca Galileo \& Martins, 2006: 26, fig. 5. Syn. nov.

Localidade-tipo: Bolívia, Cochabamba: km 95 estrada de Cochabamba a Villa Tunari $(1800 \mathrm{~m})$

O exame do exemplar e das descrições e figuras permitiu propor a presente sinonímia e nos parece adequado manter a espécie no gênero Acanthoderes.

Material examinado. BOLÍVIA, Chaco: macho.

\section{Acanthoderes (Symperasmus) thoracica White, 1855}

Acanthoderes thoracicus White, 1855: 359.

Localidade-tipo: Brasil, Pará.

Psapharochus thoracicus; Aurivillius, 1908: 10

Acanthoderes (Psapharochus) thoracica; Aurivillius, 1923: 388 (cat.). Symperasmus thoracicus; Tavakilian et al., 1997: 308, 315, 339.

Acanthoderes (Symperasmus) thoracica; Monné, 2001: 38 (cat. hosts); 2005b: 165 (cat.).

Acanthoderes (Psapharochrus) uyapensis Martins \& Galileo, 2003: 616, fig. 2. Syn. nov.

Localidade-tipo: Bolívia, La Paz: Uyapi (Guanay).

Psapharochrus uyapensis; Monné, 2005b: 214 (cat.).

O exame de diversos exemplares e a comparação entre as descrições e as fotografias dos holótipos possibilitou a sinonímia proposta.

Distribuição: Guiana Francesa, Brasil (Pará, Rondônia, Mato Grosso), Peru, Bolívia.

Material examinado. BRASIL, Amazonas: Benjamin Constant, fêmea, X.1958, B. Sampaio col.; Borba, Lago Acará, fêmea, VIII.1943, A. Parko col.; Manaus, fêmea, III.1959, C. Elias col.; Parintins, fêmea, 1940; Tabatinga, macho, VI.1957, L. G. Pereira col.; Tefé: macho, I.1957, R. Carvalho col.; Pará: Acari, fêmea, 9.IX.1955, N. L. Cerqueira col.; Jacareacanga, macho, XII.1968, M. Alvarenga col.; Óbidos, macho, I.1956, F. M. Oliveira col. Tucuruí, macho, I.1980, B. Silva col.; Rondônia: Ouro Preto do Oeste, macho, X.1983, Roppa, Becker \& Silva col.; Vilhena, fêmea, XI.1973, Alvarenga \& Roppa col.; Mato Grosso: Diamantino, Alto Rio Arinos, fêmea, X.1983, B. Silva col. Sinop, macho, fêmea, X.1974, Alvarenga \& Roppa col.. PERU, Junin: Chanchamayo, fêmea, 10.II.1949, H. Zellibor col.; Sátipo, macho, XI.1937; Loreto: confluência rios Zumun e Yahuasyacu, macho, I.1980, Becker col.

\section{Irundisaua Martins \& Galileo, 2005}

Irundisaua Martins \& Galileo, 2005: 15; Monné, 2005b: 176 (cat.). Espécie-tipo: Irundisaua ocularis Martins \& Galileo, 2005 (designação original).

Natagaima Lane, 1972: 367; Julio, 2003: 75 (transf., chave spp.); Monné, 2005b: 181 (cat.).

Espécie-tipo: Natagaima balteata Lane, 1972 (designação original).

Lane (1972) descreveu Natagaima na tribo Anisocerini. Julio (2003) transferiu o gênero para Acanthoderini e descreveu duas espécies. Martins \& Galileo (2005) descreveram Irundisaua em Acanthoderini, sem perceber a transferência realizada por Julio (op. cit.).

Irundisaua Martins \& Galileo, 2005 é considerado sinônimo de Natagaima Lane, 1972 porque apresenta os mesmos caracteres, inclusive o antenômero IV achatado e acentuadamente alargado na metade apical. Resulta, entretanto, que Natagaima Lane, 1972 está preocupado por Natagaima Beier, 1960 (Orthoptera, Tettigoniidae), sendo assim, propomos a substituição pelo sinônimo júnior Irundisaua Martins \& Galileo, 2005.

Irundisaua balteata (Lane, 1972) comb. nov.

Natagaima balteata Lane, 1972: 368, fig. 2; Julio, 2003: 77, figs. 1-3; Monné, 2005b: 181 (cat.).

Localidade-tipo: Colômbia, Natagaima. Distribuição: Colômbia.

Irundisaua heloisae (Julio, 2003) comb. nov.

Natagaima heloisae Julio, 2003: 80, fig. 3; Monné, 2005b: 181 (cat.). Localidade-tipo: Equador, Napo: Coca. Distribuição: Equador.

Irundisaua moacyri (Julio, 2003) comb. nov.

Natagaima moacyri Julio, 2003: 78, fig. 2.; Monné, 2005b: 181 (cat.). Localidade-tipo: Colômbia, Nariño: Tumaco. Distribuição: Colômbia.

\section{Irundisaua ocularis Martins \& Galileo, 2005}

Irundisaua ocularis Martins \& Galileo, 2005: 15, figs. 11, 12; Monné, 2005b: 177 (cat.).

Localidade-tipo: Colômbia, Boyaca: Santa Maria. Distribuição: Colômbia

Agradecimentos. Ao Prof. Ubirajara R. Martins (MZSP) pela confirmação das sinonímias em Acanthoderini.

\section{REFERENNCIAS}

Aurivillius, C. 1908. Cerambyciden aus den Granzgebieten zwischen Peru und Bolivien gesammelt von Dr. Nils Holmgren. Arkiv för Zoologi 5: 1-13.

Aurivillius, C. 1921. Neue Cerambyciden aus der Sammlung G. van Roon. Tijdschrift voor Entomologie 64: 46-53.

Aurivillius, C. 1923. Coleopterorum Catalogus, pars 74, Cerambycidae: Lamiinae. Berlin, W. Junk, p. 323-704.

Di Iorio, O. 1998. One new species, new records and biology of Lissonotini (Coleoptera, Cerambycidae) from Argentina and Bolivia. Giornale Italiano di Entomologia 9: 27-36.

Di Iorio, O. 2002. Nomenclatural notes for some American Cerambycidae (Coleoptera). Giornale Italiano di Entomologia 10: $73-76$.

Galileo, M. H. M. \& U. R. Martins. 2006. Novos táxons em Hippopsini, Desmiphorini, Xenofreini e Acanthoderini (Coleoptera, Cerambycidae, Lamiinae). Papéis Avulsos de Zoologia 46: 21 29.

Julio, C. E. A. 2003. Revisão do gênero Natagaima (Coleoptera, Cerambycidae, Lamiinae). Iheringia, Zoologia 93: 75-80.

Lane, F. 1972. Cerambycoidea Neotropica nova VIII (Coleoptera). Studia Entomologica 15: 352-382.

Lane, F. 1973. Cerambycoidea Neotropica nova IX (Coleoptera). Studia Entomologica 16: 371-438.

Martins, U.R. \& M. H. M. Galileo. 2003. Novas espécies e nota sobre Lamiinae (Coleoptera, Cerambycidae) neotropicais da coleção Arriagada, Santiago, Chile. Revista Brasileira de Entomologia 47: 615-620

Martins, U. R. \& M. H. M. Galileo. 2005. Cerambycidae (Coleoptera) da Colômbia. VII. Novos táxons, novos registros, nova sinonímia, 
nova combinação e novo nome. Revista Brasileira de Zoologia 22: $5-18$

Monné, M. A. 2001. Catalogue of the Neotropical Cerambycidae (Coleoptera) with known host plant - Part III: Subfamily Lamiinae, Tribes Acanthocinini to Apomecynini. Publicações Avulsas do Museu Nacional 92: 1-94.

Monné, M. A. 2005a. Catalogue of the Cerambycidae (Coleoptera) of the Neotropical Region. Part I. Subfamily Cerambycinae. Zootaxa 946: $1-765$.

Monné, M. A. 2005b. Catalogue of the Cerambycidae (Coleoptera) of the Neotropical Region. Part II. Subfamily Lamiinae. Zootaxa 1023: $1-759$.

Monné, M.A. 2006. Catalogue of the Cerambycidae (Coleoptera) of the Neotropical Region. Part III. Subfamilies Parandrinae, Prioninae, Anoplodermatinae, Aseminae, Spondylidinae, Lepturinae, Oxypeltinae, and addenda to the Cerambycinae and Lamiinae. Zootaxa 1212: 1-244.
Monné, M. L. \& M. A. Monné. 1998. Sinopse do gênero Tropidosoma Perty, 1832 e novas sinonímias em Trachyderini (Coleoptera, Cerambycidae, Cerambycinae). Revista Brasileira de Entomologia 41: 531-534.

Tavakilian, G. L.; A. Berkov; B. Meurer-Grimes \& S. Mori. 1997. Neotropical tree species and their faunas of xylophagous longicorns (Coleoptera: Cerambycidae) in French Guiana. The Botanical Review 63: 304-355.

Viana, M. J. \& A. Martínez. 1992. Un nuevo género y espécie de Lissonotini (Col., Cerambycidae, Cerambycinae). Anales de la Sociedad Científica Argentina 222: 27-31.

White, A. 1855. Catalogue of the coleopterous insects in the collection of the British Museum. Longicornia 2. London, British Museum, 8: 175-412.

Recebido em 17/05/2007; aceito em 08/10/2007 